\title{
Simulating ADAS Sensors, their Placement and Environment
}

\author{
Jaroslav Rymus, technical consultant \\ ESI Group, \\ Pilsen, Czech Republic \\ Jaroslav.Rymus@esi-group.com
}

\begin{abstract}
With the advent of autonomous vehicles, carmakers are increasingly involved in developments of Active Safety and Advanced Driver Assistance Systems. Those systems are part of complex electronic equipment, that - with its sensors, microcontrollers, cable harnesses - will represent up to $40 \%$ of the total manufacturing cost. Today, virtual prototyping is integral part of development process, assuring fast innovation cycles and minimizing costs. A multiscale hybrid strategy is proposed to simulate Advanced Driver Assistance Systems (ADAS) in both Short Radar Range (SRR) and Long Radar Range (LRR). While SRR devices (typically blind spot / dead-angle radars) are operating at $24 \mathrm{GHz}$, the LRR devices used for Adaptive Cruise Control systems are utilizing the $77 \mathrm{GHz}$ band. The sensor itself is modeled using FDTD or MoM/MLFMM methods. Then, the related electromagnetic environment is computed by means of Physical Optics (PO). Dedicated PO upgrades are utilized to handle plastic parts (bumper, front grille) in front of a sensor.
\end{abstract}

Keywords: virtual prototyping, simulations, computational electromagnetics, sensor, ADAS

References:

[1] CEM One at ESI Group official site: https://www.esi-group.com/software-solutions/virtualenvironment/electromagnetics/cem-one-solution.

[2] Virtual Prototyping at ESI Group official site: https://www.esi-group.com/smart-virtualprototyping.

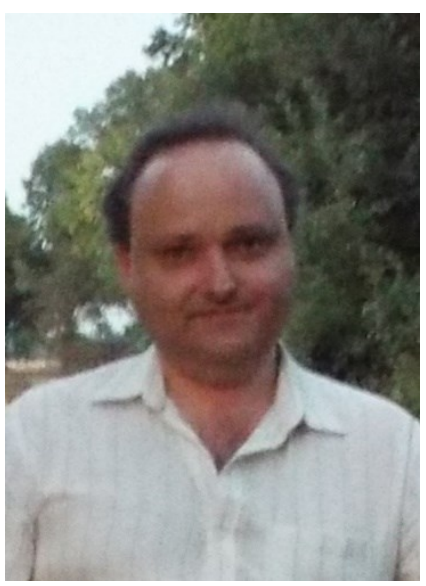

Jaroslav Rymus is with MECAS ESI, the Czech subsidiary of ESI Group. Jaroslav is involved in development of dedicated modules for VisualCEM, a part of ESI's virtual prototyping platform also including CEM One, a complete solution for computational electromagnetics. His current professional interests include antenna optimization, antenna matching as well as use of characteristic modes for antenna design.

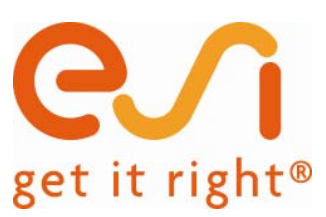

